Mathematical Research Letters 1, 601-611 (1994)

\title{
BOGOMOLOV UNSTABILITY ON ARITHMETIC SURFACES
}

\author{
ATsushi MoRIWAKI
}

\begin{abstract}
A в STRACт. In this paper, we will consider an arithmetic analogue of Bogomolov unstability theorem, i.e. if $(E, h)$ is a torsion free Hermitian sheaf on an arithmetic surface $X$ and $\widehat{\operatorname{deg}}\left((\operatorname{rk} E-1) \widehat{c}_{1}(E, h)^{2}-(2 \operatorname{rk} E) \widehat{c}_{2}(E, h)\right)>$ 0 , then there is a non-zero saturated subsheaf $F$ of $E$ such that the point $\widehat{c}_{1}\left(F,\left.h\right|_{F}\right) / \mathrm{rk} F-\widehat{c}_{1}(E, h) / \mathrm{rk} E$ lies in the positive cone of $X$.
\end{abstract}

\section{Introduction}

In [Bo], Bogomolov proved an unstability theorem, namely, if a vector bundle $E$ on a complex projective surface $S$ satisfies an inequality (rk $E$ 1) $c_{1}(E)^{2}-(2 \mathrm{rk} E) c_{2}(E)>0$, then there is a saturated subsheaf $F$ of $E$ such that $c_{1}(F) / \mathrm{rk} F-c_{1}(E) / \mathrm{rk} E$ belongs to the positive cone of $S$. In this paper, we would like to consider an arithmetic analogue of Bogomolov unstability theorem.

Let $f: X \rightarrow \operatorname{Spec}\left(O_{K}\right)$ be a regular arithmetic surface over the ring of integers of a number field $K$ with $f_{*} \mathcal{O}_{X}=O_{K}$, and $\operatorname{deg}_{K}: \widehat{\mathrm{CH}}^{1}(X)_{\mathbb{R}} \rightarrow \mathbb{R}$ the natural homomorphism given by

$$
\operatorname{deg}_{K}: \widehat{\mathrm{CH}}^{1}(X)_{\mathbb{R}} \stackrel{z}{\longrightarrow} \mathrm{CH}^{1}(X)_{\mathbb{R}} \stackrel{\otimes K}{\longrightarrow} \mathrm{CH}^{1}\left(X_{K}\right)_{\mathbb{R}} \stackrel{\operatorname{deg}}{\longrightarrow} \mathbb{R} .
$$

The positive cone $\widehat{C}_{++}(X)$ of $X$ is defined by the set of all elements $x \in$ $\widehat{\mathrm{CH}}^{1}(X)_{\mathbb{R}}$ with $\widehat{\operatorname{deg}}\left(x^{2}\right)>0$ and $\operatorname{deg}_{K}(x)>0$. A torsion free Hermitian sheaf $(E, h)$ on $X$ is said to be arithmetically unstable if there is a non-zero saturated subsheaf $F$ of $E$ with

$$
\frac{\widehat{c}_{1}\left(F,\left.h\right|_{F}\right)}{\operatorname{rk} F}-\frac{\widehat{c}_{1}(E, h)}{\operatorname{rk} E} \in \widehat{C}_{++}(X),
$$

where $\left.h\right|_{F}$ is the Hermitian metric of $F$ given by the restriction of $h$ to $F$. The main theorem of this paper is the following.

Received June 17, 1994. 
Theorem A. If $(E, h)$ is a torsion free Hermitian sheaf on $X$ and

$$
\widehat{\operatorname{deg}}\left((\operatorname{rk} E-1) \widehat{c}_{1}(E, h)^{2}-(2 \operatorname{rk} E) \widehat{c}_{2}(E, h)\right)>0,
$$

then $(E, h)$ is arithmetically unstable.

Let $A$ be an element of $\widehat{\mathrm{CH}}^{1}(X)_{\mathbb{R}}$ with $\widehat{\operatorname{deg}}(A \cdot x)>0$ for all $x \in \widehat{C}_{++}(X)$, i.e. according to terminology in $\S 1.1, A$ is an element of the weak positive cone $\widehat{C}_{+}(X)$. A torsion free Hermitian sheaf $(E, h)$ on $X$ is said to be arithmetically $\mu$-semistable with respect to $A$ if, for all non-zero saturated subsheaves $F$ of $E$,

$$
\frac{\widehat{\operatorname{deg}}\left(\widehat{c}_{1}\left(F,\left.h\right|_{F}\right) \cdot A\right)}{\operatorname{rk} F} \leq \frac{\widehat{\operatorname{deg}}\left(\widehat{c}_{1}(E, h) \cdot A\right)}{\operatorname{rk} E} .
$$

Under the above terminology, we have the following corollary of Theorem A.

Corollary B. If $(E, h)$ is arithmetically $\mu$-semistable with respect to $A$, then

$$
\widehat{\operatorname{deg}}\left((2 \operatorname{rk} E) \widehat{c}_{2}(E, h)-(\operatorname{rk} E-1) \widehat{c}_{1}(E, h)^{2}\right) \geq 0 .
$$

If $A=\left(0, \sum_{\sigma \in K(\mathbb{C})} 1 /[K: \mathbb{Q}]\right)$, then arithmetic $\mu$-semistability of $(E, h)$ with respect to $A$ is nothing more than $\mu$-semistability of $E_{\overline{\mathbb{Q}}}$. So this corollary gives a generalization of [Mo1], [Mo2] and [So].

In $\S 1$, we will prepare several basic facts of the positive cone and Hermitian vector spaces. In $\S 2$, we will consider finiteness of saturated subsheaves in a Hermitian vector bundle, which will be crucial for the proof of Theorem A. $\S 3$ is devoted to the proof of Theorem A and Corollary B.

\section{Preliminaries}

1.1. Positive cone of arithmetic Chow group. Here, we consider basic properties of the positive cone of the arithmetic Chow group of codimension 1.

Let $K$ be a number field and $O_{K}$ the ring of integers of $K$. Let $f$ : $X \rightarrow \operatorname{Spec}\left(O_{K}\right)$ be a regular arithmetic surface with $f_{*} \mathcal{O}_{X}=O_{K}$, and $\operatorname{deg}_{K}: \widehat{\mathrm{CH}}^{1}(X)_{\mathbb{R}} \rightarrow \mathbb{R}$ the natural homomorphism defined by

$$
\operatorname{deg}_{K}: \widehat{\mathrm{CH}}^{1}(X)_{\mathbb{R}} \stackrel{z}{\longrightarrow} \mathrm{CH}^{1}(X)_{\mathbb{R}} \stackrel{\otimes K}{\longrightarrow} \mathrm{CH}^{1}\left(X_{K}\right)_{\mathbb{R}} \stackrel{\operatorname{deg}}{\longrightarrow} \mathbb{R} .
$$

We set

$$
\begin{aligned}
\widehat{C}_{++}(X) & =\left\{x \in \widehat{\mathrm{CH}}^{1}(X)_{\mathbb{R}} \mid \widehat{\operatorname{deg}}\left(x^{2}\right)>0 \text { and } \operatorname{deg}_{K}(x)>0\right\} \text { and } \\
\widehat{C}_{+}(X) & =\left\{x \in \widehat{\mathrm{CH}}^{1}(X)_{\mathbb{R}} \mid \widehat{\operatorname{deg}}(x \cdot y)>0 \text { for all } y \in \widehat{C}_{++}(X)\right\} .
\end{aligned}
$$

$\widehat{C}_{++}(X)$ (resp. $\left.\widehat{C}_{+}(X)\right)$ is called the positive cone of $X$ (resp. the weak positive cone of $X$ ). First of all, we have the following lemma. 
Lemma 1.1.1. (1.1.1.1) If $h \in \widehat{C}_{++}(X), x \in \widehat{\mathrm{CH}}^{1}(X)_{\mathbb{R}}$ and $\widehat{\operatorname{deg}}(x \cdot h)=0$, then $\widehat{\operatorname{deg}}\left(x^{2}\right) \leq 0$.

(1.1.1.2) If $x \in \widehat{\mathrm{CH}}^{1}(X)_{\mathbb{R}}, \widehat{\operatorname{deg}}\left(x^{2}\right) \geq 0$ and $\operatorname{deg}_{K}(x)>0$, then $x \in$ $\widehat{C}_{+}(X)$.

(1.1.1.3) If $h \in \widehat{C}_{++}(X), x \in \widehat{\mathrm{CH}}^{1}(X)_{\mathbb{R}}, \widehat{\operatorname{deg}}\left(x^{2}\right) \geq 0$ and $\widehat{\operatorname{deg}}(x \cdot h)>0$, then $x \in \widehat{C}_{+}(X)$.

Proof. (1.1.1.1) Let $t$ be a real number with $\operatorname{deg}_{K}(x-t h)=0$. Then, by Hodge index theorem (cf. [Fa], [Hr] or [Mo3]), $\widehat{\operatorname{deg}}\left((x-t h)^{2}\right) \leq 0$. Thus, $\widehat{\operatorname{deg}}\left(x^{2}\right)+t^{2} \widehat{\operatorname{deg}}\left(h^{2}\right) \leq 0$. Therefore, $\widehat{\operatorname{deg}}\left(x^{2}\right) \leq 0$.

(1.1.1.2) Let $y \in \widehat{C}_{++}(X)$ and $t$ a real number with $\operatorname{deg}_{K}(y-t x)=0$. Then, $t>0$. By Hodge index theorem, $\widehat{\operatorname{deg}}\left((y-t x)^{2}\right) \leq 0$. Thus, we have

$$
\widehat{\operatorname{deg}}\left(y^{2}\right)+t^{2} \widehat{\operatorname{deg}}\left(x^{2}\right) \leq 2 t \widehat{\operatorname{deg}}(x \cdot y) .
$$

Therefore, $\widehat{\operatorname{deg}}(x \cdot y)>0$. Hence, $x \in \widehat{C}_{+}(X)$.

(1.1.1.3) Let $y \in \widehat{C}_{++}(X)$ and $t$ a real number with $\widehat{\operatorname{deg}}(y-t x \cdot h)=0$. Then, $t>0$ by (1.1.1.2). (1.1.1.1) implies $\widehat{\operatorname{deg}}\left((y-t x)^{2}\right) \leq 0$. Thus, by the same way as above, we have $\widehat{\operatorname{deg}}(x \cdot y)>0$, which says $x \in \widehat{C}_{+}(X)$.

$\widehat{C}_{++}(X)$ and $\widehat{C}_{+}(X)$ have the following properties.

Proposition 1.1.2. (1.1.2.1) $\widehat{C}_{++}(X) \subset \widehat{C}_{+}(X)$.

(1.1.2.2) If $x, y \in \widehat{C}_{++}(X)$ and $t>0$, then $x+y, t x \in \widehat{C}_{++}(X)$.

(1.1.2.3) If $x, y \in \widehat{C}_{+}(X)$ and $t>0$, then $x+y, t x \in \widehat{C}_{+}(X)$.

(1.1.2.4) $\widehat{C}_{++}(X)=\left\{x \in \widehat{\mathrm{CH}}^{1}(X)_{\mathbb{R}} \mid \widehat{\operatorname{deg}}(x \cdot y)>0 \forall y \in \widehat{C}_{+}(X)\right\}$.

Proof. (1.1.2.1) and (1.1.2.2) are straightforward from (1.1.1.2). (1.1.2.3) is obvious.

(1.1.2.4) Clearly, we have

$$
\widehat{C}_{++}(X) \subseteq\left\{x \in \widehat{\mathrm{CH}}^{1}(X)_{\mathbb{R}} \mid \widehat{\operatorname{deg}}(x \cdot y)>0 \text { for all } y \in \widehat{C}_{+}(X)\right\} .
$$

We assume that $\widehat{\operatorname{deg}}(x \cdot y)>0$ for all $y \in \widehat{C}_{+}(X)$. If we set $B=$ $\left(0, \sum_{\sigma \in K(\mathbb{C})} 1 /[K: \mathbb{Q}]\right)$, then $B \in \widehat{C}_{+}(X)$. Thus, $\operatorname{deg}_{K}(x)=\widehat{\operatorname{deg}}(x \cdot B)>$ 0 . Hence, it is sufficient to show $\widehat{\operatorname{deg}}\left(x^{2}\right)>0$. Here, we fix $h \in \widehat{C}_{++}(X)$ with $\widehat{\operatorname{deg}}\left(h^{2}\right)=1$. We set $t=\widehat{\operatorname{deg}}(x \cdot h)>0$. Since $\widehat{\operatorname{deg}}(x-t h \cdot h)=0$, by (1.1.1.1), $\widehat{\operatorname{deg}}\left((x-t h)^{2}\right) \leq 0$. If $\widehat{\operatorname{deg}}\left((x-t h)^{2}\right)=0$, then

$$
\widehat{\operatorname{deg}}\left(x^{2}\right)=\widehat{\operatorname{deg}}\left((t h+(x-t h))^{2}\right)=t^{2}>0 .
$$


Thus, we may assume that $\widehat{\operatorname{deg}}\left((x-t h)^{2}\right)$ is negative. If we set $s=$ $\left(-\widehat{\operatorname{deg}}\left((x-t h)^{2}\right)\right)^{1 / 2}$ and $l=(x-t h) / s$, then $x=t h+s l, \widehat{\operatorname{deg}}\left(l^{2}\right)=-1$ and $\widehat{\operatorname{deg}}(h \cdot l)=0$. Let us consider $y=h+l$. Since $\widehat{\operatorname{deg}}\left(y^{2}\right)=0$ and $\widehat{\operatorname{deg}}(y \cdot h)=1$, by (1.1.1.3), $y \in \widehat{C}_{+}(X)$. Thus, $\widehat{\operatorname{deg}}(x \cdot y)=t-s>0$. Therefore

$$
\widehat{\operatorname{deg}}\left(x^{2}\right)=t^{2}-s^{2}=(t+s)(t-s)>0 .
$$

Hence, $x \in \widehat{C}_{++}(X)$.

Finally we consider the following proposition.

Proposition 1.1.3. For $z \in \widehat{\mathrm{CH}}^{1}(X)_{\mathbb{R}}$, we set

$$
W(z)=\left\{u \in \widehat{C}_{+}(X) \mid \widehat{\operatorname{deg}}(z \cdot u)>0\right\} .
$$

If $x \notin \widehat{C}_{++}(X), y \in \widehat{C}_{++}(X)$ and $W(x) \neq \emptyset$, then $W(x) \varsubsetneqq W(x+y)$.

Proof. Clearly, $W(x) \subseteq W(x+y)$. By virtue of (1.1.2.4), $W(x) \varsubsetneqq \widehat{C}_{+}(X)$. Let $u_{1} \in W(x), u_{2} \in \widehat{C}_{+}(X) \backslash W(x)$ and $t=-\widehat{\operatorname{deg}}\left(x \cdot u_{2}\right) / \widehat{\operatorname{deg}}\left(x \cdot u_{1}\right)$. Then, $t \geq 0$ and $\widehat{\operatorname{deg}}\left(x \cdot u_{2}+t u_{1}\right)=0$. Hence, $u_{2}+t u_{1} \notin W(x)$. On the other hand, by (1.1.2.3), $u_{2}+t u_{1} \in \widehat{C}_{+}(X)$. Moreover, $\widehat{\operatorname{deg}}\left(y \cdot u_{2}+t u_{1}\right)>0$. Thus, $\widehat{\operatorname{deg}}\left(x+y \cdot u_{2}+t u_{1}\right)>0$. Therefore $u_{2}+t u_{1} \in W(x+y)$.

1.2. Hermitian vector space. Let $V$ be a $\mathbb{C}$-vector space, $h_{V}$ a Hermitian metric on $V$ and $W$ a subvector space of $V$. Considering the restriction of $h$ to $W$, the metric $h_{V}$ induces a metric $h_{W}$ of $W$, which is called the submetric of $W$ induced by $h_{V}$. Let $W^{\perp}$ be the orthogonal complement of $W$. Then the natural homomorphism $W^{\perp} \longrightarrow V / W$ is isomorphic. Thus we have a metric $h_{V / W}$ of $V / W$ given by $\left.h\right|_{W^{\perp}}$. This metric is called the quotient metric of $V / W$ induced by $h_{V}$.

Proposition 1.2.1. Let $\left(V, h_{V}\right)$ be a Hermitian vector space over $\mathbb{C}$ and $U, W$ subspaces of $V$ with $U \subset W$. Let $h_{W}=\left.\left(h_{V}\right)\right|_{W}$ and $h_{V / U}$ the quotient metric of $V / U$ induced by $h_{V}$. We consider two Hermitian metrics of $W / U$. Let $h_{s}=\left.\left(h_{V / U}\right)\right|_{W / U}$ and $h_{q}$ the quotient metric induced by $h_{W}$. Then, we have $h_{s}=h_{q}$.

Proof. Let $V=U \oplus U^{\perp}$ be the orthogonal decomposition of $V$ and $f$ : $U^{\perp} \rightarrow V / U$ the natural isomorphism. Then, for $x, y \in W / U, h_{s}(x, y)=$ $h_{V}\left(f^{-1}(x), f^{-1}(y)\right)$.

Let $W=U \oplus U_{W}^{\perp}$ be the orthogonal decomposition of $W$, i.e. $U_{W}^{\perp}$ is the orthogonal complement of $U$ in $W$, and $g: U_{W}^{\perp} \rightarrow W / U$ the natural isomorphism. Then, $h_{q}(x, y)=h_{V}\left(g^{-1}(x), g^{-1}(y)\right)$ for $x, y \in W / U$. 
On the other hand, $U_{W}^{\perp} \subset U^{\perp}$ and the following diagram is commutative.

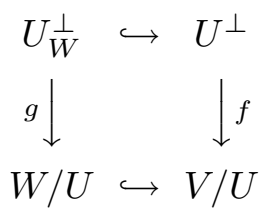

Thus, we have $h_{s}=h_{q}$.

\section{Finiteness of saturated subsheaves}

In this section, we will consider finiteness of saturated subsheaves in a Hermitian vector bundle, which will be crucial for the proof of Theorem A.

Theorem 2.1. Let $K$ be a number field, and $O_{K}$ the ring of integers of $K$. Let $f: X \rightarrow \operatorname{Spec}\left(O_{K}\right)$ be a regular arithmetic surface with $f_{*} \mathcal{O}_{X}=O_{K}$, $(E, h)$ a Hermitian vector bundle on $X$, and $(H, k)$ a Hermitian line bundle on $X$. If $H_{K}$ is ample, then, for constants $C_{1}$ and $C_{2}$, the set

$$
\mathcal{F}=\left\{\begin{array}{l|l}
L & \begin{array}{l}
L \text { is a rank-1 saturated subsheaf of } E \text { with } \\
\widehat{\operatorname{deg}}\left(\widehat{c}_{1}\left(L,\left.h\right|_{L}\right) \cdot \widehat{c}_{1}(H, k)\right) \geq C_{1} \text { and } \operatorname{deg}\left(L_{K}\right) \geq C_{2}
\end{array}
\end{array}\right\}
$$

is finite.

Proof. First of all, we need the following Lemma.

Lemma 2.1.1. Let $K$ be an infinite field, $C$ a smooth projective curve over $K$, and $E$ a vector bundle on $C$ of rank $r \geq 2$. Then, for every real number $M$, there is a rank-1 saturated subsheaf $L$ of $E$ with $\operatorname{deg}(L)<M$.

Proof. Let $H$ be an ample line bundle on $X$, and let $n$ be a positive integer such that $n \operatorname{deg}(H)>-M$ and $E \otimes H^{n}$ is generated by global sections. Let us consider the following closed set $\Sigma$ in $C \times \mathbb{P}\left(H^{0}\left(C, E \otimes H^{n}\right)\right)$;

$$
\Sigma=\left\{(x, s) \in C \times \mathbb{P}\left(H^{0}\left(C, E \otimes H^{n}\right)\right) \mid s(x)=0\right\} .
$$

Let $p: \Sigma \rightarrow C$ be the natural projection. Since $E \otimes H^{n}$ is generated by global sections, $\operatorname{codim}\left(p^{-1}(x), \mathbb{P}\left(H^{0}\left(C, E \otimes H^{n}\right)\right)\right)=r$. Therefore, $\operatorname{codim}\left(\Sigma, C \times \mathbb{P}\left(H^{0}\left(C, E \otimes H^{n}\right)\right)\right)=r$ and $\operatorname{dim} \Sigma<\operatorname{dim} \mathbb{P}\left(H^{0}\left(C, E \otimes H^{n}\right)\right)$. Thus, the natural projection $q: \Sigma \rightarrow \mathbb{P}\left(H^{0}\left(C, E \otimes H^{n}\right)\right)$ is not surjective. Hence, since $\#(K)$ is infinite,

$$
(p(\Sigma))(K) \varsubsetneqq \mathbb{P}\left(H^{0}\left(C, E \otimes H^{n}\right)\right)(K) .
$$


Thus, there is a section $s \in H^{0}\left(C, E \otimes H^{n}\right)$ with $\operatorname{div}(s)=\emptyset$, which induces an injective homomorphism $H^{-n} \rightarrow E$. Since $\operatorname{div}(s)=\emptyset$, the image of $H^{-n} \rightarrow E$ is saturated.

Let us start the proof of Theorem 2.1. Clearly we may assume $r=$ rk $E \geq 2$. By Lemma 2.1.1, there is a filtration : $\{0\}=F_{0} \subset F_{1} \subset \cdots \subset$ $F_{r-1} \subset F_{r}=E$ such that

(2.1.2) $F_{i} / F_{i-1}$ is a rank-1 torsion free sheaf for every $1 \leq i \leq r$.

(2.1.3) $\operatorname{deg}\left(\left(F_{i} / F_{i-1}\right)_{K}\right)<C_{2}$ for $1 \leq i \leq r-1$.

Here we claim

Claim 2.1.4. If $L$ is a line bundle on $X$ with $\operatorname{deg}\left(L_{K}\right) \geq C_{2}$, and $\varphi: L \rightarrow$ $F_{r-1}$ is a homomorphism, then $\varphi=0$.

Proof. We assume that $\varphi \neq 0$. Choose $i$ in such a way that $\varphi(L) \subseteq$ $F_{i}$ and $\varphi(L) \nsubseteq F_{i-1}$. Then, we have an injective homomorphism $L \rightarrow$ $F_{i} / F_{i-1}$. Since $i \leq r-1, \operatorname{deg}\left(L_{K}\right) \geq C_{2}>\operatorname{deg}\left(\left(F_{i} / F_{i-1}\right)_{K}\right)$. This is a contradiction.

Let $Q$ be the double dual of $E / F_{r-1}$ and $h_{Q}$ the quotient metric of $Q$ induced by $h$ via $E \rightarrow E / F_{r-1}$, i.e. $h_{Q}=\left.h\right|_{F^{\perp}}$. Pick up $L \in \mathcal{F}$. By Claim 2.1.4, we have the natural injection $L \rightarrow Q$. So there is an effective divisor $D_{L}$ on $X$ such that $L \otimes \mathcal{O}_{X}\left(D_{L}\right) \simeq Q$. Let $D_{L}^{h}$ (resp. $D_{L}^{v}$ ) be the horizontal part of $D_{L}$ (resp. the vertical part of $D_{L}$ ). Then, we have

Claim 2.1.5. If $D_{L}^{h}=D_{L^{\prime}}^{h}$ for $L, L^{\prime} \in \mathcal{F}$, then $L=L^{\prime}$.

Proof. Let us consider $M=L \otimes \mathcal{O}_{X}\left(-D_{L^{\prime}}^{v}\right)$ and $M^{\prime}=L^{\prime} \otimes \mathcal{O}_{X}\left(-D_{L}^{v}\right)$. Since $D_{L}^{h}=D_{L^{\prime}}^{h}, M$ and $M^{\prime}$ have the same image in $Q$ via $E \rightarrow Q$. Therefore, we have $M+F_{r-1}=M^{\prime}+F_{r-1}$. Moreover, since $M \rightarrow Q$ and $M^{\prime} \rightarrow Q$ are injective, $M \cap F_{r-1}=M^{\prime} \cap F_{r-1}=\{0\}$. Hence, we have a homomorphism $M \rightarrow M^{\prime} \oplus F_{r-1} \rightarrow F_{r-1}$, which must be zero by Claim 2.1.4. Thus $M \subseteq M^{\prime}$. By the same way, $M^{\prime} \subseteq M$. Hence, $M=M^{\prime}$. So $L=L^{\prime}$ because $L$ is the saturation of $M$ in $E$ and $L^{\prime}$ is the saturation of $M^{\prime}$ in $E$.

We set $C_{3}=\widehat{\operatorname{deg}}\left(\widehat{c}_{1}\left(Q, h_{Q}\right) \cdot \widehat{c}_{1}(H, k)\right)-C_{1}$ and $C_{4}=\operatorname{deg}\left(Q_{K}\right)-C_{2}$. Let $D_{L}^{h}=\sum_{i} a_{i} \Gamma_{i}$ be the irreducible decomposition of $D_{L}^{h}$. Then, we have

Claim 2.1.6. $\sum_{i} a_{i} \widehat{\operatorname{deg}}\left(\widehat{c}_{1}\left(\left.(H, k)\right|_{\Gamma_{i}}\right)\right) \leq C_{3}$ and $\sum_{i} a_{i}\left[K\left(\Gamma_{i}\right): K\right] \leq C_{4}$.

Proof. Since $L \otimes \mathcal{O}_{X}\left(D_{L}\right) \simeq Q$, we have $\operatorname{deg}\left(L_{K}\right)+\operatorname{deg}\left(\left(D_{L}\right)_{K}\right)=\operatorname{deg}\left(Q_{K}\right)$. Thus we get $\sum_{i} a_{i}\left[K\left(\Gamma_{i}\right): K\right] \leq C_{4}$.

We choose a Hermitian metric $h_{D_{L}}$ of $\mathcal{O}_{X}\left(D_{L}\right)$ in such a way that $\left(L,\left.h\right|_{L}\right) \otimes\left(\mathcal{O}_{X}\left(D_{L}\right), h_{D_{L}}\right)$ is isometric to $\left(Q, h_{Q}\right)$. Let 1 be the canonical section of $H^{0}\left(X, \mathcal{O}_{X}\left(D_{L}\right)\right)$ with $\operatorname{div}(1)=D_{L}$, and $D_{L}^{v}=\sum_{j} b_{j} l_{j}$ the 
irreducible decomposition of the vertical part of $D_{L}$. Then, we have

$$
\begin{aligned}
\widehat{\operatorname{deg}}\left(\widehat{c}_{1}\left(\mathcal{O}_{X}\left(D_{L}\right), h_{D_{L}}\right) \cdot \widehat{c}_{1}(H, k)\right) & =\sum_{i} a_{i} \widehat{\operatorname{deg}}\left(\widehat{c}_{1}\left(\left.(H, k)\right|_{\Gamma_{i}}\right)\right) \\
& +\sum_{j} b_{j} \operatorname{deg}\left(\left.H\right|_{l_{j}}\right)-\frac{1}{2} \sum_{\sigma \in K(\mathbb{C})} \int_{X_{\sigma}} \log \left(h_{D_{L}}(1,1)\right) c_{1}\left(H_{\sigma}, k_{\sigma}\right) .
\end{aligned}
$$

Since $h_{Q}$ is a quotient metric of $h$, we can see $h_{D_{L}}(1,1) \leq 1$ for all points of each infinite fiber $X_{\sigma}$. Therefore, we get

$$
\begin{aligned}
\sum_{i} a_{i} \widehat{\operatorname{deg}}\left(\widehat{c}_{1}\left(\left.(H, k)\right|_{\Gamma_{i}}\right)\right) \leq \widehat{\operatorname{deg}}\left(\widehat{c}_{1}\left(\mathcal{O}_{X}\left(D_{L}\right), h_{D_{L}}\right) \cdot \widehat{c}_{1}(H, k)\right) \\
=\widehat{\operatorname{deg}}\left(\widehat{c}_{1}\left(Q, h_{Q}\right) \cdot \widehat{c}_{1}(H, k)\right)-\widehat{\operatorname{deg}}\left(\widehat{c}_{1}\left(L,\left.h\right|_{L}\right) \cdot \widehat{c}_{1}(H, k)\right) \leq C_{3} .
\end{aligned}
$$

Thus, we obtain our claim.

To complete our theorem, by Claim 2.1.5, it is sufficient to see that $\left\{D_{L}^{h} \mid L \in \mathcal{F}\right\}$ is finite. Since $\sum_{i} a_{i}\left[K\left(\Gamma_{i}\right): K\right] \leq C_{4}$, we have $a_{i} \leq C_{4}$ and $\left[\mathbb{Q}\left(\Gamma_{i}\right): \mathbb{Q}\right] \leq C_{4}[K: \mathbb{Q}]$. Let $D_{L}^{h}=D_{L}^{h}(+)+D_{L}^{h}(-)$ be the decomposition of $D_{L}^{h}$ such that

$$
D_{L}^{h}(+)=\sum_{\operatorname{deg}\left(c_{1}\left(\left.(H, k)\right|_{\Gamma_{i}}\right)\right)>0} a_{i} \Gamma_{i} \text { and } D_{L}^{h}(-)=\sum_{\operatorname{deg}\left(c_{1}\left(\left.(H, k)\right|_{\Gamma_{i}}\right)\right) \leq 0} a_{i} \Gamma_{i} .
$$

By Northcott's theorem (cf. Theorem 2.6 in Chapter 2 of [La]), the set $\left\{D_{L}^{h}(-) \mid L \in \mathcal{F}\right\}$ is finite. Hence, there is a constant $C_{5}$ depending only on $\mathcal{F}$ with

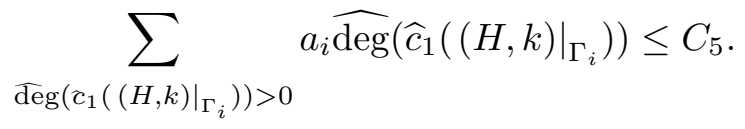

Thus, $\left\{D_{L}^{h}(+) \mid L \in \mathcal{F}\right\}$ is finite. Therefore, $\left\{D_{L}^{h} \mid L \in \mathcal{F}\right\}$ is finite.

Corollary 2.2. Let $K$ be a number field, and $O_{K}$ the ring of integers of $K$. Let $f: X \rightarrow \operatorname{Spec}\left(O_{K}\right)$ be a regular arithmetic surface with $f_{*} \mathcal{O}_{X}=O_{K}$, $(E, h)$ a Hermitian vector bundle on $X$, and $(H, k)$ a Hermitian line bundle on $X$. If $H_{K}$ is ample, then, for constants $C_{1}$ and $C_{2}$, the set

$$
\left\{\begin{array}{l|l}
\widehat{c}_{1}\left(F,\left.h\right|_{F}\right) \in \widehat{\mathrm{CH}}^{1}(X) & \begin{array}{l}
F \text { is a non-zero saturated subsheaf of } E \text { with } \\
\widehat{\operatorname{deg}}\left(\widehat{c}_{1}\left(F,\left.h\right|_{F}\right) \cdot \widehat{c}_{1}(H, k)\right) \geq C_{1} \\
\text { and } \operatorname{deg}\left(F_{K}\right) \geq C_{2}
\end{array}
\end{array}\right\}
$$

is finite.

Proof. Since $\operatorname{det} F$ is a saturated subsheaf of $\bigwedge^{\text {rk } F} E$, our corollary is an immediate consequence of Theorem 2.1. 


\section{Proof of Bogomolov unstability (Theorem A)}

Before the proof of Theorem A, we will fix notations. Let $K$ be a number field and $O_{K}$ the ring of integers of $K$. Let $f: X \rightarrow \operatorname{Spec}\left(O_{K}\right)$ be a regular arithmetic surface with $f_{*} \mathcal{O}_{X}=O_{K}$. Let $\left(F, h_{F}\right)$ and $\left(E, h_{E}\right)$ be torsion free Hermitian sheaves on $X$. We set

$$
\widehat{\delta}\left(E, h_{E}\right)=\widehat{\operatorname{deg}}\left(\frac{\operatorname{rk} E-1}{2 \operatorname{rk} E} \widehat{c}_{1}\left(E, h_{E}\right)^{2}-\widehat{c}_{2}\left(E, h_{E}\right)\right) .
$$

and

$$
\widehat{d}\left(\left(F, h_{F}\right),\left(E, h_{E}\right)\right)=\frac{\widehat{c}_{1}\left(F, h_{F}\right)}{\operatorname{rk} F}-\frac{\widehat{c}_{1}\left(E, h_{E}\right)}{\operatorname{rk} E} .
$$

Then, we have the following formula.

Lemma 3.1. Let $0 \rightarrow\left(S, h_{S}\right) \rightarrow\left(E, h_{E}\right) \rightarrow\left(Q, h_{Q}\right) \rightarrow 0$ be an exact sequence of torsion free Hermitian sheaves on $X$ such that $h_{S}$ and $h_{Q}$ are the induced metric by $h_{E}$. Then, we have

$$
\widehat{\delta}\left(E, h_{E}\right) \leq \widehat{\delta}\left(S, h_{S}\right)+\widehat{\delta}\left(Q, h_{Q}\right)+\frac{(\operatorname{rk} E)(\operatorname{rk} S)}{2 \operatorname{rk} Q} \widehat{\operatorname{deg}}\left(\widehat{d}\left(\left(S, h_{S}\right),\left(E, h_{E}\right)\right)^{2}\right) .
$$

Proof. First of all, $\widehat{c}_{1}\left(E, h_{E}\right)=\widehat{c}_{1}\left(S, h_{S}\right)+\widehat{c}_{1}\left(Q, h_{Q}\right)$. Moreover, by Proposition 7.3 of [Mo1],

$$
\widehat{\operatorname{deg}}\left(\widehat{c}_{2}\left(E, h_{E}\right)-\widehat{c}_{2}\left(\left(S, h_{S}\right) \oplus\left(Q, h_{Q}\right)\right)\right) \geq 0 .
$$

Thus, by an easy calculation, we have our lemma.

Let us start the proof of Theorem A. Let $E^{\vee \vee}$ be the double dual of $E$. Then, $\widehat{c}_{2}\left(E^{\vee \vee}, h\right)=\widehat{c}_{2}(E, h)-\log \left(\#\left(E^{\vee \vee} / E\right)\right)$. So we may assume that $E$ is locally free. First we claim

Claim 3.2. There is a non-zero saturated subsheaf $F$ of $E$ such that

$$
\frac{\operatorname{deg}\left(F_{K}\right)}{\operatorname{rk} F}-\frac{\operatorname{deg}\left(E_{K}\right)}{\operatorname{rk} E}>0
$$

Proof. Since $\widehat{\operatorname{deg}}\left((\operatorname{rk} E-1) \widehat{c}_{1}(E, h)^{2}-(2 \operatorname{rk} E) \widehat{c}_{2}(E, h)\right)>0$, by the main theorem of [Mo1], $E_{\overline{\mathbb{Q}}}$ is not semistable. Let $F^{\prime}$ be the maximal destabilizing sheaf of $E_{\overline{\mathbb{Q}}}$. For $\tau \in \operatorname{Gal}(\overline{\mathbb{Q}} / K)$, let us consider $\tau\left(F^{\prime}\right)$. Then, $\tau\left(F^{\prime}\right) \subset E_{\overline{\mathbb{Q}}}, \operatorname{deg}\left(\tau\left(F^{\prime}\right)\right)=\operatorname{deg}\left(F^{\prime}\right)$ and $\operatorname{rk} \tau\left(F^{\prime}\right)=\operatorname{rk} F^{\prime}$, which means that $\tau\left(F^{\prime}\right)$ is also a maximal destabilizing sheaf of $E_{\overline{\mathbb{Q}}}$. Thus, by the uniqueness 
of the maximal destabilizing sheaf, we have $\tau\left(F^{\prime}\right)=F^{\prime}$. Therefore, $F^{\prime}$ is defined over $K$. Hence, there is a saturated subsheaf $F$ of $E$ with $F_{K}=F^{\prime}$. Thus, we have our claim.

Let $(H, k)$ be a Hermitian line bundle on $X$ such that $H_{K}$ is ample. Since

$$
\begin{array}{r}
\widehat{\operatorname{deg}}\left(\widehat{d}\left(\left(F,\left.h\right|_{F}\right),(E, h)\right) \cdot \widehat{c}_{1}(H, c k)\right)=\widehat{\operatorname{deg}}\left(\widehat{d}\left(\left(F,\left.h\right|_{F}\right),(E, h)\right) \cdot \widehat{c}_{1}(H, k)\right) \\
-\frac{\log (c)[K: \mathbb{Q}]}{2}\left(\frac{\operatorname{deg}\left(F_{K}\right)}{\operatorname{rk} F}-\frac{\operatorname{deg}\left(E_{K}\right)}{\operatorname{rk} E}\right)
\end{array}
$$

and $\widehat{\operatorname{deg}}\left(\widehat{c}_{1}(H, c k)^{2}\right)=\widehat{\operatorname{deg}}\left(\widehat{c}_{1}(H, k)^{2}\right)-\log (c)[K: \mathbb{Q}] \operatorname{deg}\left(H_{K}\right)$, we may assume that $\left.\widehat{\operatorname{deg}}\left(\widehat{d}\left(\left(F,\left.h\right|_{F}\right),(E, h)\right)\right) \cdot \widehat{c}_{1}(H, k)\right)>0$ and $\widehat{c}_{1}(H, k) \in \widehat{C}_{++}(X)$ if we replace $k$ by $c k$ with sufficiently small positive number $c$. Here we consider the following set.

$$
\mathcal{G}=\left\{\begin{array}{l|l}
G & \begin{array}{l}
G \text { is a non-zero saturated subsheaf of } E \text { with } \\
\widehat{\operatorname{deg}}\left(\widehat{d}\left(\left(G,\left.h\right|_{G}\right),(E, h)\right) \cdot \widehat{c}_{1}(H, k)\right)>0 \text { and } \\
\operatorname{deg}_{K}\left(\widehat{d}\left(\left(G,\left.h\right|_{G}\right),(E, h)\right)\right)>0
\end{array}
\end{array}\right\} .
$$

Then, $F \in \mathcal{G}$. Moreover, by Corollary 2.2,

$$
\left\{\widehat{d}\left(\left(G,\left.h\right|_{G}\right),(E, h)\right) \in \widehat{\mathrm{CH}}^{1}(X)_{\mathbb{Q}} \mid G \in \mathcal{G}\right\}
$$

is finite.

We will prove Theorem A by induction on $\mathrm{rk} E$.

Claim 3.3. If $\operatorname{rk} E=2$, then $\widehat{d}\left(\left(F,\left.h\right|_{F}\right),(E, h)\right) \in \widehat{C}_{++}(X)$.

Proof. Let $h_{E / F}$ be the quotient metric of $E / F$, i.e. $h_{E / F}=\left.h\right|_{F^{\perp}}$. By Lemma 3.1,

$\widehat{\delta}(E, h) \leq \widehat{\delta}\left(F,\left.h\right|_{F}\right)+\widehat{\delta}\left(E / F, h_{E / F}\right)+\frac{(\operatorname{rk} E)(\operatorname{rk} F)}{2 \operatorname{rk} E / F} \widehat{\operatorname{deg}}\left(\widehat{d}\left(\left(F,\left.h\right|_{F}\right),(E, h)\right)^{2}\right)$.

Since $\operatorname{rk} F=\operatorname{rk} E / F=1, \widehat{\delta}\left(F,\left.h\right|_{F}\right) \leq 0$ and $\widehat{\delta}\left(E / F, h_{E / F}\right) \leq 0$. Therefore,

$$
\widehat{\operatorname{deg}}\left(\widehat{d}\left(\left(F,\left.h\right|_{F}\right),(E, h)\right)^{2}\right)>0 .
$$

Thus, $\widehat{d}\left(\left(F,\left.h\right|_{F}\right),(E, h)\right) \in \widehat{C}_{++}(X)$.

From now on, we assume rk $E \geq 3$. As in (1.1.3.1), for $x \in \widehat{\mathrm{CH}}^{1}(X)_{\mathbb{R}}$, we set

$$
W(x)=\left\{u \in \widehat{C}_{+}(X) \mid \widehat{\operatorname{deg}}(x \cdot u)>0\right\} .
$$

Here we claim 
Claim 3.4. Under the hypothesis of induction, if $\widehat{\operatorname{deg}}\left(\widehat{d}\left(\left(G,\left.h\right|_{G}\right),(E, h)\right)^{2}\right)$ $\leq 0$ for $G \in \mathcal{G}$, then there is $G_{1} \in \mathcal{G}$ with

$$
W\left(\widehat{d}\left(\left(G,\left.h\right|_{G}\right),(E, h)\right)\right) \varsubsetneqq W\left(\widehat{d}\left(\left(G_{1},\left.h\right|_{G_{1}}\right),(E, h)\right)\right) .
$$

Proof. We set $h_{E / G}=\left.h\right|_{G^{\perp}}$. First of all, by Lemma 3.1,

$$
\begin{aligned}
\widehat{\delta}(E, h) \leq \widehat{\delta}\left(G,\left.h\right|_{G}\right)+\widehat{\delta}(E / G & \left., h_{E / G}\right) \\
& +\frac{(\operatorname{rk} E)(\operatorname{rk} G)}{2 \operatorname{rk} E / G} \widehat{\operatorname{deg}}\left(\widehat{d}\left(\left(G,\left.h\right|_{G}\right),(E, h)\right)^{2}\right) .
\end{aligned}
$$

Since $\widehat{\delta}(E, h)>0$ and $\widehat{\operatorname{deg}}\left(\widehat{d}\left(\left(G,\left.h\right|_{G}\right),(E, h)\right)^{2}\right) \leq 0$, we have either that $\widehat{\delta}\left(G,\left.h\right|_{G}\right)>0$ or that $\widehat{\delta}\left(E / G, h_{E / G}\right)>0$.

If $\widehat{\delta}\left(G,\left.h\right|_{G}\right)>0$, then by hypothesis of induction there is a non-zero saturated subsheaf $G_{1}$ of $G$ with $\widehat{d}\left(\left(G_{1},\left.h\right|_{G_{1}}\right),\left(G,\left.h\right|_{G}\right)\right) \in \widehat{C}_{++}(X)$. Here since

$$
\widehat{d}\left(\left(G_{1},\left.h\right|_{G_{1}}\right),(E, h)\right)=\widehat{d}\left(\left(G_{1},\left.h\right|_{G_{1}}\right),\left(G, h_{G}\right)\right)+\widehat{d}\left(\left(G,\left.h\right|_{G}\right),(E, h)\right),
$$

we have $G_{1} \in \mathcal{G}$. Moreover, by Proposition 1.1.3, we get

$$
W\left(\widehat{d}\left(\left(G,\left.h\right|_{G}\right),(E, h)\right)\right) \varsubsetneqq W\left(\widehat{d}\left(\left(G_{1},\left.h\right|_{G_{1}}\right),(E, h)\right)\right) .
$$

If $\widehat{\delta}\left(E / G, h_{E / G}\right)>0$, then by hypothesis of induction there is a nonzero saturated subsheaf $T$ of $E / G$ such that $\widehat{d}\left(\left(T,\left.h_{E / G}\right|_{T}\right),\left(E / G, h_{E / G}\right)\right) \in$ $\widehat{C}_{++}(X)$. Take a saturated subsheaf $G_{1}$ in $E$ with $G \subset G_{1}$ and $G_{1} / G=T$. Let $h_{G_{1} / G}$ be the induced quotient metric by $h_{G_{1}}$. By Proposition 1.2.1, we have $\left.h_{E / G}\right|_{T}=h_{G_{1} / G}$. Thus, by an easy calculation, we get

$$
\begin{aligned}
\widehat{d}\left(\left(G_{1},\left.h\right|_{G_{1}}\right),(E, h)\right)=\frac{\operatorname{rk}\left(G_{1} / G\right)}{\operatorname{rk} G_{1}} & \widehat{d}\left(\left(T,\left.h_{E / G}\right|_{T}\right),\left(E / G, h_{E / G}\right)\right) \\
& +\frac{\operatorname{rk} G \operatorname{rk}\left(E / G_{1}\right)}{\operatorname{rk} G_{1} \operatorname{rk}(E / G)} \widehat{d}\left(\left(G,\left.h\right|_{G}\right),\left(E, h_{E}\right)\right) .
\end{aligned}
$$

Therefore, $G_{1} \in \mathcal{G}$, and by Proposition 1.1.3,

$$
W\left(\widehat{d}\left(\left(G,\left.h\right|_{G}\right),(E, h)\right)\right) \varsubsetneqq W\left(\widehat{d}\left(\left(G_{1},\left.h\right|_{G_{1}}\right),(E, h)\right)\right) .
$$

Hence we get Claim 3.4. 
Here we assume that $\widehat{\operatorname{deg}}\left(\widehat{d}\left(\left(G,\left.h\right|_{G}\right),(E, h)\right)^{2}\right) \leq 0$ for all $G \in \mathcal{G}$. Then, since $F \in \mathcal{G}$, by Claim 3.4, $\exists$ a sequence $\left\{G_{0}=F, G_{1}, G_{2}, \ldots, G_{n}, \ldots\right\}$ in $\mathcal{G}$ such that

$$
W\left(\widehat{d}\left(\left(G_{i},\left.h\right|_{G_{i}}\right),(E, h)\right)\right) \varsubsetneqq W\left(\widehat{d}\left(\left(G_{j},\left.h\right|_{G_{j}}\right),(E, h)\right)\right)
$$

for all $i<j$. In particular, $\widehat{d}\left(\left(G_{i},\left.h\right|_{G_{i}}\right),(E, h)\right)$ gives distinct elements in $\widehat{\mathrm{CH}}^{1}(X)_{\mathbb{Q}}$. On the other hand,

$$
\left\{\widehat{d}\left(\left(G,\left.h\right|_{G}\right),(E, h)\right) \in \widehat{\mathrm{CH}}^{1}(X)_{\mathbb{Q}} \mid G \in \mathcal{G}\right\}
$$

is finite. This is a contradiction. Thus, to get our theorem, we note that there is $G \in \mathcal{G}$ with $\widehat{\operatorname{deg}}\left(\widehat{d}\left(\left(G,\left.h\right|_{G}\right),(E, h)\right)^{2}\right)>0$.

3.5 Proof of Corollary B. Finally, we give the proof of Corollary B. We assume that

$$
\widehat{\operatorname{deg}}\left((2 \operatorname{rk} E) \widehat{c}_{2}(E, h)-(\operatorname{rk} E-1) \widehat{c}_{1}(E, h)^{2}\right)<0 .
$$

Then, by Theorem A, there is a non-zero saturated subsheaf $F$ of $E$ with

$$
\frac{\widehat{c}_{1}\left(F,\left.h\right|_{F}\right)}{\mathrm{rk} F}-\frac{\widehat{c}_{1}(E, h)}{\mathrm{rk} E} \in \widehat{C}_{++}(X) .
$$

Thus,

$$
\frac{\widehat{\operatorname{deg}}\left(\widehat{c}_{1}\left(F,\left.h\right|_{F}\right) \cdot A\right)}{\operatorname{rk} F}-\frac{\widehat{\operatorname{deg}}\left(\widehat{c}_{1}(E, h) \cdot A\right)}{\operatorname{rk} E}>0 .
$$

This is a contradiction.

\section{References}

[Bo] F. A. Bogomolov, Holomorphic tensors and vector bundles on projective varieties, Math. USSR-Izv. 13 (1978), 499-555.

[Fa] G. Faltings, Calculus on arithmetic surfaces, Ann. of Math. 119 (1984), 387-424.

[Hr] P. Hriljac, Heights and Arakelov's intersection theory, Amer. J. Math. 107 (1985), 23-38.

[La] S. Lang, Fundamentals of Diophantine Geometry, Springer-Verlag, 1983.

[Mo1] A. Moriwaki, Inequality of Bogolomov-Gieseker type on arithmetic surfaces, Duke Math. J. 74 (1994), 713-761.

[Mo2] A. Moriwaki, Arithmetic Bogomolov-Gieseker's inequality, to appear, Amer. J. Math..

[Mo3] A. Moriwaki, Hodge index theorem for arithmetic cycles of codimension one, Algebraic geometry e-prints (alg-geom@publications.math.duke.edu), \#9403011.

[So] C. Soulé, A vanishing theorem on arithmetic surfaces, Invent. 116 (1994), 577599 .

Department of Mathematics, Faculty of Science, Kroto Univerity, KyO TO, 606-01, JAPAN.

Current address: Department of Mathematics, University of California, Los Angeles, 405 Hilgard Avenue, Los Angeles, California 90024.

E-mail address: moriwaki@math.ucla.edu 